\title{
Swift and XMM-Newton observations of the dark GRB 050326
}

\author{
A. Moretti ${ }^{1}$, A. De Luca ${ }^{2}$, D. Malesani ${ }^{3}$, S. Campana ${ }^{1}$, A. Tiengo ${ }^{2}$, A. Cucchiara ${ }^{4}$, J. N. Reeves ${ }^{5}$, G. Chincarini ${ }^{1,6}$, \\ C. Pagani ${ }^{1,4}$, P. Romano ${ }^{1}$, G. Tagliaferri ${ }^{1}$, P. Banat ${ }^{1}$, M. Capalbi ${ }^{7}$, M. Perri ${ }^{7}$, G. Cusumano ${ }^{8}$, V. Mangano ${ }^{8}$, T. Mineo ${ }^{8}$, \\ V. La Parola ${ }^{8}$, A. Beardmore ${ }^{9}$, M. Goad ${ }^{9}$, J. P. Osborne ${ }^{9}$, J. E. Hill ${ }^{5,10}$, L. Angelini ${ }^{5,11}$, D. N. Burrows ${ }^{4}$, \\ S. Kobayashi ${ }^{4}$, P. Mészáros ${ }^{4}$, B. Zhang ${ }^{12}$, S. D. Barthelmy ${ }^{5}$, L. Barbier ${ }^{5}$, N. E. White ${ }^{5}$, \\ E. E. Fenimore ${ }^{13}$, L. R. Cominsky ${ }^{14}$, and N. Gehrels ${ }^{5}$
}

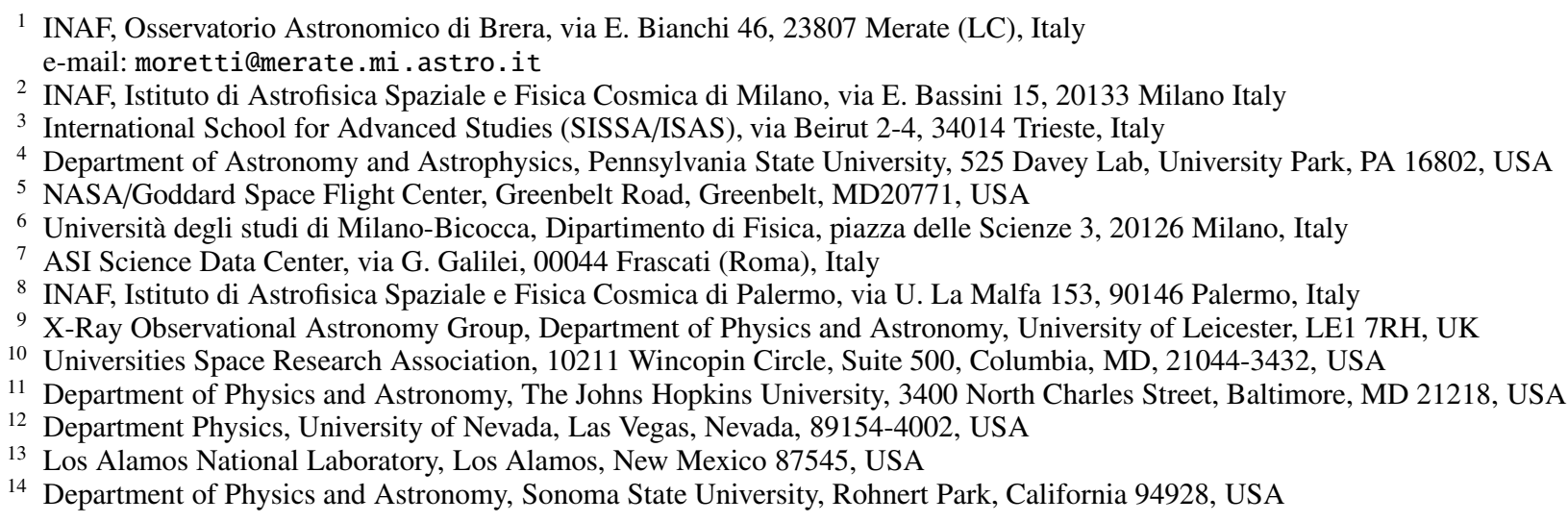

Received 26 July 2005 / Accepted 14 December 2005

ABSTRACT

\begin{abstract}
We present Swift and XMM-Newton observations of the bright gamma-ray burst GRB 050326, detected by the Swift Burst Alert Telescope. The Swift X-Ray Telescope (XRT) and XMM-Newton discovered the X-ray afterglow beginning $54 \mathrm{~min}$ and $8.5 \mathrm{~h}$ after the burst, respectively. The prompt GRB 050326 fluence was $(7.7 \pm 0.9) \times 10^{-6} \mathrm{erg} \mathrm{cm}^{-2}(20-150 \mathrm{keV})$, and its spectrum was hard, with a power law photon index $\Gamma=1.25 \pm 0.03$. The X-ray afterglow was quite bright, with a flux of $7 \times 10^{-11} \mathrm{erg} \mathrm{cm}^{-2} \mathrm{~s}^{-1}(0.3-8 \mathrm{keV})$, $1 \mathrm{~h}$ after the burst. Its light curve did not show any break nor flares between $\sim 1 \mathrm{~h}$ and $\sim 6 \mathrm{~d}$ after the burst, and decayed with a slope $\alpha=1.70 \pm 0.05$. The afterglow spectrum is well fitted by a power-law model, suffering absorption both in the Milky Way and in the host galaxy. The rest-frame hydrogen column density is significant, $N_{\mathrm{H}, z} \gtrsim 4 \times 10^{21} \mathrm{~cm}^{-2}$, and the redshift of the absorber was constrained to be $z>1.5$. There was good agreement between the spatial, temporal, and spectral parameters as derived by SwiftXRT and XMM-Newton. By comparing the prompt and afterglow fluxes, we found that an early break probably occurred before the beginning of the XRT observation, similarly to many other cases observed by Swift. However, the properties of the GRB 050326 afterglow are well described by a spherical fireball expanding in a uniform external medium, so a further steepening is expected at later times. The lack of such a break allowed us to constrain the jet half-opening angle $\vartheta_{\mathrm{j}} \gtrsim 7^{\circ}$. Using the redshift constraints provided by the X-ray analysis, we also estimated that the beaming-corrected gamma-ray energy was larger than $3 \times 10^{51} \mathrm{erg}$, at the high end of GRB energies. Despite the brightness in X rays, only deep limits could be placed by Swift-UVOT at optical and ultraviolet wavelengths. Thus, this GRB was a "truly dark" event, with the optical-to-X-ray spectrum violating the synchrotron limit. The optical and X-ray observations are therefore consistent either with an absorbed event or with a high-redshift one. To obey the Ghirlanda relation, a moderate/large redshift $z \gtrsim 4.5$ is required.
\end{abstract}

Key words. gamma rays: bursts - gamma rays: observations - X-rays: bursts

\section{Introduction}

The Swift satellite (Gehrels et al. 2004) is a mission dedicated to the study of gamma-ray bursts (GRBs) and their afterglows. GRBs are detected and localized by the Burst Alert Telescope (BAT, Barthelmy et al. 2005), and followed up at X-ray (0.2-10 keV) and optical/ultraviolet (1700-6000 $⿱$ ) wavelengths by the X-Ray Telescope (XRT, Burrows et al. 2005a) and the Ultraviolet/Optical Telescope (UVOT, Roming et al. 2005a). During the first year of operation, Swift has observed some 75 GRB afterglows, already doubling the pre-Swift sample. This rich dataset has allowed to study in detail the X-ray light curves, both at early and late times, leading to the discovery of a complex behaviour (e.g Tagliaferri et al. 2005a; Nousek et al. 2005; Chincarini et al. 2005; Cusumano et al. 2006a). Coupled with optical data, either from UVOT (e.g. Blustin et al. 2006) or ground-based observatories (e.g. Berger et al. 2005), this has opened a new era in the afterglow modeling. Swift also provided the first detection of truly dark GRBs, that is, events with no optical emission up to very deep limits (Roming et al. 2005b). The study of high-redshift GRBs has also started, with the discovery of the first burst at $z>6$ (Watson et al. 2005b; Cusumano et al. 2006b; Haislip et al. 2006; Price et al. 2005; Tagliaferri et al. 2005b; Kawai et al. 2006). Moreover, it was found that Swift 
GRBs have a larger average redshift than those discovered by earlier missions (Jakobsson et al. 2006).

During the performance verification and calibration phase (2004 Nov. 20 through 2005 Apr. 5), Swift observed sixteen GRB afterglows. Twelve of them were observed in automatic mode, and, among these, eight could be promptly (within 200 s since the trigger) observed by XRT and UVOT. In the remaining four cases, the beginning of the observation was delayed by approximately $50 \mathrm{~min}$ due to the Earth occultation constraints. This is the case for the bright GRB 050326, which was discovered by BAT on 2005 Mar. 26 at 9:53:55 UT (Markwardt et al. 2005). Its coordinates were $\alpha_{\mathrm{J} 2000}=00^{\mathrm{h}} 27^{\mathrm{m}} 34^{\mathrm{s}}, \delta_{\mathrm{J} 2000}=-71^{\circ} 22^{\prime} 34^{\prime \prime}$, with an uncertainty radius of $3^{\prime}(95 \%$ containment, Cummings et al. 2005). This burst was also detected by the Wind-Konus experiment (Golenteskii et al. 2005), leading to the characterization of its broad-band gamma-ray spectrum.

The Swift narrow field instruments could begin observing only 54 min after the BAT trigger. A bright, uncatalogued X-ray source was detected by XRT inside the BAT error circle, and was proposed to be the X-ray afterglow (Moretti et al. 2005a). However, no source was detected by UVOT at this location (Holland et al. 2005). XRT collected data up to $6.15 \mathrm{~d}$ after the burst. Subsequently, the decay of the light curve prevented any further detection of the afterglow. This object was also observed for $45.8 \mathrm{ks}$ by XMM-Newton (Ehle \& Perez Martinez 2005; De Luca et al. 2005a), starting $8.5 \mathrm{~h}$ after the trigger.

Only limited ground-based follow-up was reported for this burst. This was likely due to its unfavorable location in the sky (very few telescopes can point at such low declination), as well as to the brightness of the Moon (which was $99 \%$ full at the time of the GRB explosion). No counterpart at wavelengths other than the $\mathrm{X}$ rays was reported.

In this work, we present a complete discussion of the Swift and XMM-Newton observations of GRB 050326. In Sect. 2 we describe the properties of the prompt emission. In Sect. 3 we describe in detail the XRT observations, the data reduction procedure, and the temporal and spectral analysis; in Sect. 4 we do the same for the XMM-Newton data. In Sect. 5 we compare the results of the two instruments. In Sect. 6 we describe the UVOT optical observations. Finally, in Sect. 7 we present the physical implications of our observations in the framework of the standard GRB afterglow model. Our conclusions are summarized in Sect. 8.

Throughout this paper, all errors are quoted at $90 \%$ confidence level for one parameter of interest, unless otherwise specified. The reduced $\chi^{2}$ will be denoted as $\chi_{v}^{2}$, and the number of degrees of freedom with the abbreviation "d.o.f.". We follow the convention $F_{v}(v, t) \propto t^{-\alpha} v^{-\beta}$, where $\alpha$ and $\beta$ are the temporal decay slope and the spectral index, respectively. As time origin, we will adopt the BAT trigger (Markwardt et al. 2005). The photon index is $\Gamma=1+\beta$. Last, we adopt the standard "concordance" cosmology parameters, namely $\Omega_{\mathrm{m}}=0.27, \Omega_{\Lambda}=0.73$, $h_{0}=0.71$ (e.g. Spergel et al. 2003).

\section{Prompt emission}

We reduced the BAT data using the latest available release of the HEADAS software (version 1.4). The light curve in the BAT energy band (20-150 keV) presents an initial, weak peak, $9 \mathrm{~s}$ before the trigger, followed by several bright distinct peaks (Fig. 1). The $T_{90}$ and $T_{50}$ durations of the burst (that is, the time intervals in which $90 \%$ and $50 \%$ of the fluence were collected, respectively) were 29.5 and $19.3 \mathrm{~s}$, in the $20-150 \mathrm{keV}$ band, respectively. We first modeled the BAT spectrum as a single power law

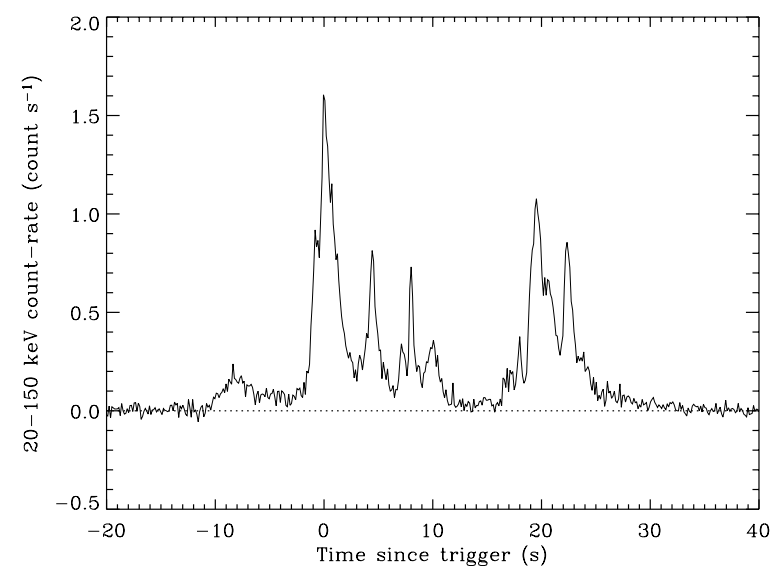

Fig. 1. The background-subtracted BAT light curve in the $20-150 \mathrm{keV}$ energy band. The origin of the time axis was set to the instrument trigger, but a weak peak is apparent $\approx 9$ s before the BAT trigger.

with photon index $\Gamma$. This provided a good fit $\left(\chi_{v}^{2}=1.06\right.$ for 53 d.o.f.), yielding $\Gamma=1.25 \pm 0.03$ in the $20-150 \mathrm{keV}$ energy range. The fluence in the same band was $(7.7 \pm 0.9) \times 10^{-6} \mathrm{erg} \mathrm{cm}^{-2}$.

GRB 050326 also triggered the Wind-Konus detector (Golenteskii et al. 2005): in the $20 \mathrm{keV}-3 \mathrm{MeV}$ energy range it lasted $38 \mathrm{~s}$, and had a fluence of $(3.22 \pm 0.05) \times 10^{-5} \mathrm{erg} \mathrm{cm}^{-2}$. Golenteskii et al. (2005) fitted the time-integrated spectrum of the burst as measured by the Wind-Konus detector with a Band model (Band et al. 1993), that is a smoothly joined broken power law with low- and high-energy photon indices $\Gamma_{1}$ and $\Gamma_{2}$, respectively, and break energy $E_{0}$. The best fit provided $\Gamma_{1}=0.74 \pm 0.09, \Gamma_{2}=2.49 \pm 0.16$, and $E_{0}=160 \pm 22 \mathrm{keV}$. The corresponding observed peak energy (that is, the energy at which the maximum of the emission is reached) was $E_{\mathrm{p}, \mathrm{obs}}=$ $\left(2-\Gamma_{1}\right) E_{0}=200 \pm 30 \mathrm{keV}$. Motivated by their results, we also performed a fit to the BAT data using the Band function. Since the break energy $E_{0}$ lies close to the upper boundary of the BAT energy range $(150 \mathrm{keV})$, we were forced to freeze $E_{0}$ and $\Gamma_{2}$ to the values determined by Wind-Konus. The fit was again good $\left(\chi_{v}^{2}=1.14\right.$ for 53 d.o.f. $)$, and provided $\Gamma_{1}=0.87 \pm 0.03$, in good agreement with the value found by Golenteskii et al. (2005). It is not surprising that both functional forms provide a good fit to the data, since they do not differ significantly inside the BAT energy range. Nevertheless, thanks to the very broad band covered by the Wind-Konus instrument, for this burst the break energy could be clearly constrained. In the following, we will consider the Band model as the best description of the GRB 050326 spectrum. With this fit, the fluence in the $20-150 \mathrm{keV}$ band was $(7.6 \pm 0.8) \times 10^{-6} \mathrm{erg} \mathrm{cm}^{-2}$. Integrating the burst spectrum from 1 to $10000 \mathrm{keV}$, we could evaluate the bolometric fluence $\mathcal{F}$ of the burst, finding $\mathcal{F}=2.4 \times 10^{-5} \mathrm{erg} \mathrm{cm}^{-2}$.

No spectral evolution could be detected in the BAT data. We split the observation in three time intervals, covering the ranges $[-9,-1],[-1,13]$, and $[13,29] \mathrm{s}$ (relative to the BAT trigger). By fitting the data with either a simple power law or with the Band model, the resulting parameters were always consistent with those derived by fitting the whole spectrum.

\section{XRT data analysis and results}

\subsection{Data reduction}

For a technical description of XRT and its operations, we refer, e.g., to Burrows et al. (2005a) and Hill et al. (2004). XRT started observing the field of GRB 050326 on 2005 Mar. 26 at 


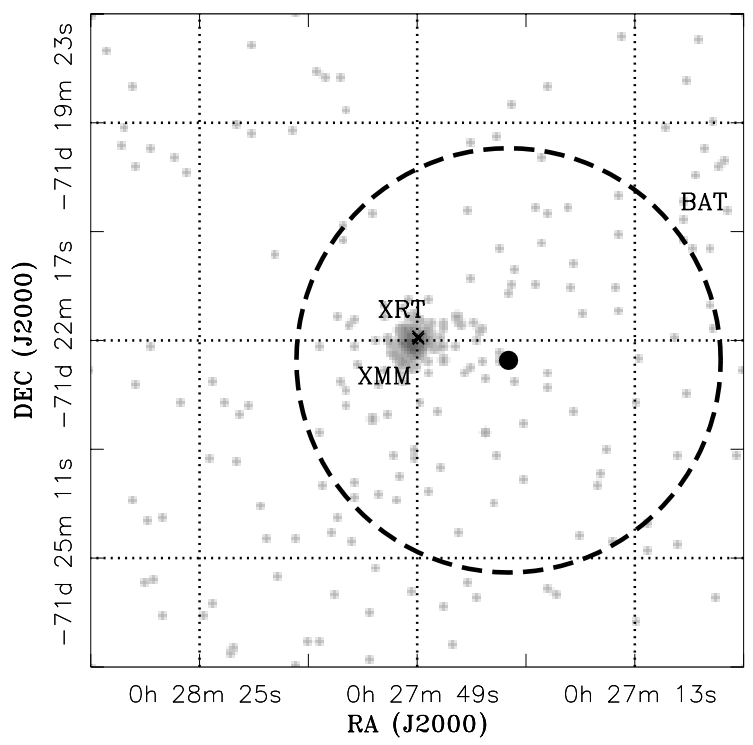

Fig. 2. XRT image of the field of GRB 050326, smoothed with a Gaussian kernel (4'.7 full width half maximum). Events were accumulated from the first segment of the observation (589 s exposure time). The BAT refined position (black dot) is also shown together with its $95 \%$ containment error circle ( $3^{\prime}$ radius, dashed line). The XRT and XMM-Newton positions (1".7 apart) are also plotted (crosses), and are almost indistinguishable. The X-ray error circles (3". 6 and 1".5 radius, respectively) are too small to be seen on this scale.

10:48:27 UT, that is, $3307 \mathrm{~s}$ after the BAT trigger. The last observation ended on 2005 Apr. 1 at 13:30:53, i.e. $6.15 \mathrm{~d}$ after the burst. Occasionally some reflected light from the Earth limb made the very low energy $(<0.2 \mathrm{keV})$ background increase significantly, so that the XRT incorrectly switched from the photon counting (PC) to the windowed timing (WT) mode, even if the target count rate was well below 1 count $\mathrm{s}^{-1}$. The effective exposure time was $59.6 \mathrm{ks}$ in PC mode and $20.7 \mathrm{ks}$ in WT mode, leading to the collection of 614 and 580 photons, respectively (0.3-10 keV energy band). As the satellite settled on the target, XRT recorded a source count rate of 1.3 count $\mathrm{s}^{-1}$, which dropped to $3 \times 10^{-4}$ count $\mathrm{s}^{-1}$ at the end of the observing campaign (2005 Apr. 1). From the third orbit after the start of the observation onwards, the source count rate was $<0.1$ count s ${ }^{-1}$, while the background level was typically $>3$ count $^{-1}$ over the whole field of view. Since WT data have only one dimensional spatial information, their $\mathrm{S} / \mathrm{N}$ ratio was much lower than that of PC mode data. We therefore decided to consider WT data only for the first two orbits, when the source $\mathrm{S} / \mathrm{N}$ was higher. Data were reduced using the xrtpipeline task of the latest available release of the HEADAS software (version 1.4). Accumulating the PC data from all observations, we found that the centroid position of the afterglow had coordinates $\alpha_{\mathrm{J} 2000}=00^{\mathrm{h}} 27^{\mathrm{m}} 49^{\prime}{ }^{\prime} 16$, $\delta_{\mathrm{J} 2000}=-71^{\circ} 22^{\prime} 14^{\prime \prime} .6$, with a $3^{\prime \prime} \cdot 6$ uncertainty radius $(95 \%$ containment, Fig. 2). This position takes into account the correction for the misalignment between the telescope and the satellite optical axis (Moretti et al. 2006). This position is 1:3 away from the refined BAT position (Cummings et al. 2005), and 3"' 4 away from the preliminary XRT position (Moretti et al. 2005a), calculated using only the data from the first orbit and without the misalignment correction.

\subsection{Temporal analysis}

In order to extract the light curve, we considered all PC data, but discarded the WT data taken after the second orbit of the XRT observation $(t>20 \mathrm{ks})$. PC events were selected having grades $0-12$ from a circle with 20 pixel radius ( $\left.47^{\prime \prime}\right)$, corresponding to $92 \%$ of the encircled energy fraction (EEF) at $1.5 \mathrm{keV}$ (Moretti et al. 2005b). Only the data in the $0.3-10 \mathrm{keV}$ band energy range were considered (even if there are no events above $7 \mathrm{keV}$ ). To take into account the pile-up effect, during the initial part of the first orbit $(t \lesssim 4000 \mathrm{~s})$ an annular extraction region with inner radius of 3 pixels ( $\left.7^{\prime \prime}\right)$ was adopted for PC data. This area includes $40 \%$ of the EEF, and the deriving PSF losses were consequently taken into account. The accuracy of the PSF model in its central part is $\sim 5 \%$ (Moretti et al. 2005b). This error was properly propagated when evaluating the final uncertainty of the PSF-corrected points in the light curve. The background in PC mode was evaluated by integrating the signal from an annulus with inner and outer radii of 50 and 90 pixel, respectively, centered at the afterglow position. Inside this region, the contamination from the afterglow is expected to be negligible. In WT mode, events were selected having grades $0-2$ from a 20 pixel (47") wide rectangular region, centered on the detector X coordinate of the afterglow. To estimate the background in WT mode, we considered a region of the same size centered 40 pixels $\left(94^{\prime \prime}\right)$ away from the center of the afterglow.

The XRT observation was split into different time segments because of the Earth occultation constraints. Each satellite orbit lasts $\approx 5800 \mathrm{~s}$, while the target could be typically observed for approximately $1000 \mathrm{~s}$ per orbit. To extract the light curve, the source events were binned in $10 \mathrm{~s}$ intervals, and these bins were further grouped to ensure a minimum of 50 counts per bin. When the counts in the last bin of each orbit were less than half of the required minimum ( 25 counts), the bin was merged with the previous one. From the fifth orbit onwards, XRT did not collect enough photons within a single orbit, so data from different orbits were merged. We eventually obtained a backgroundsubtracted light curve composed by 25 points, with a minimum of 10 and a maximum of 57 counts per bin.

The resulting light curve is shown in Fig. 3, and displays a uniform decay rate, with no indications of breaks or flares. A single power law fit provides a good description to the data, yielding a decay slope $\alpha=1.64 \pm 0.07$. In order to look for spectral variations across the observation, we computed the afterglow hardness ratio as a function of time. To this end, we selected the events with energy below and above several pivotal energies, and computed the ratio of the count rates between the energy bands. No significant variation was found over the whole observation, after setting the pivotal energy to $1,1.5$ and $2 \mathrm{keV}$.

\subsection{Spectral analysis}

To extract the spectrum of the source in WT mode, we used the same extraction regions, the same background regions, and the same screening as for the temporal analysis. For PC mode, however, we further selected only grade $0-4$ events in order to improve the spectral resolution. The spectrum was binned in order to ensure a minimum of 20 counts per energy bin, ignoring channels below $0.3 \mathrm{keV}$. The spectral analysis was performed using XSPEC (v11.3). We first considered WT and PC data separately, using the former for the first 2 orbits ( 3 to $15 \mathrm{ks}$ after the burst) and the latter for the rest of the observation. In both cases, the spectrum was fitted with an absorbed power-law model, yielding good $\chi^{2}$ values (Table 1 ). The best-fit values for the hydrogen 


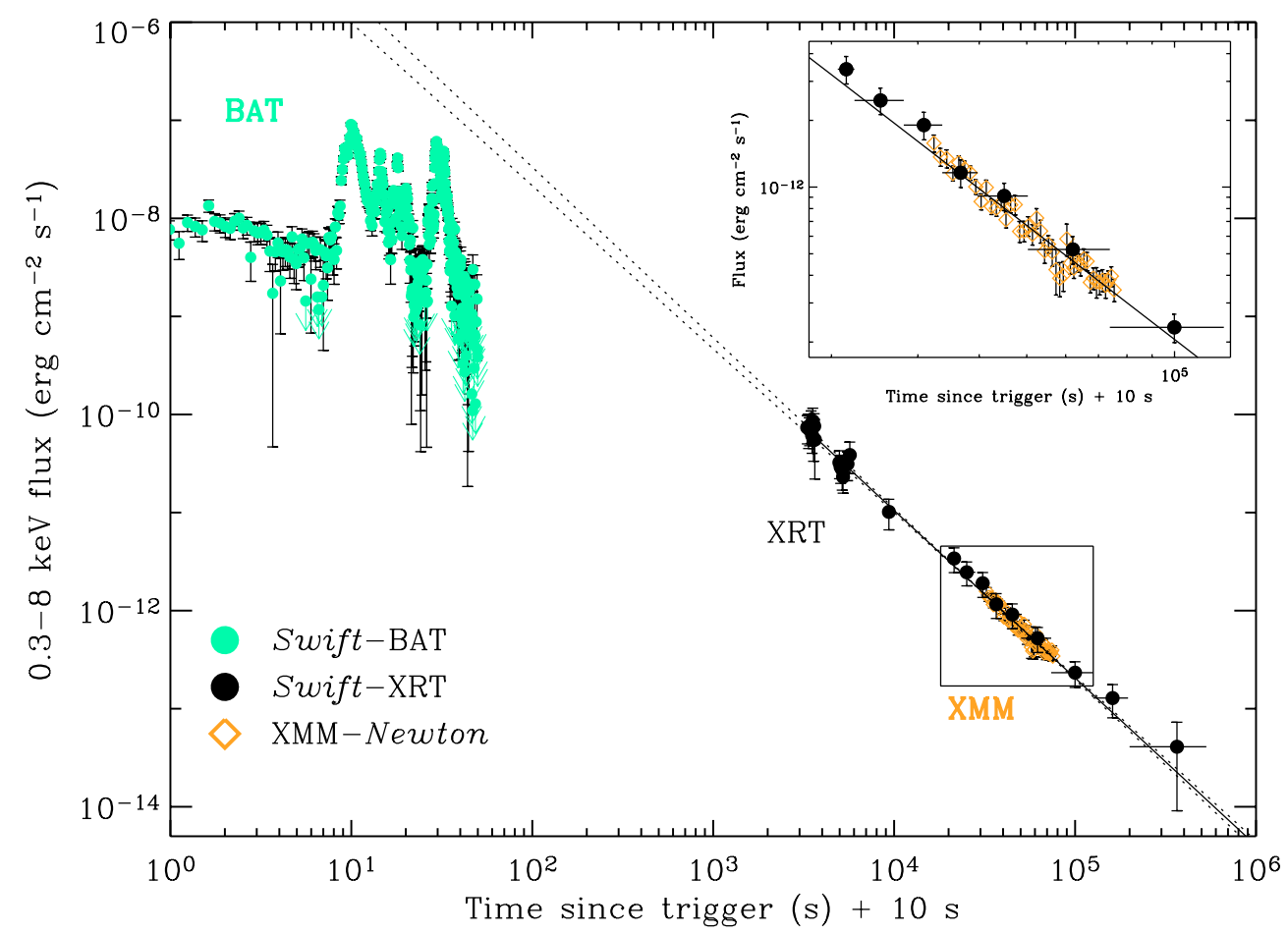

Fig. 3. The light curve of GRB 050326 and of its afterglow in the $0.3-8 \mathrm{keV}$ energy band (see text for the computation of the flux conversion factors). XRT (black circles) and XMM-Newton data (empty diamonds) show a very good agreement (see also the inset). The solid line shows the fit to the combined XRT/XMM afterglow light curve. The dotted lines indicate the $90 \%$ errors of the extrapolated X-ray light curve. Light filled circles indicate the extrapolation of the BAT data to the $0.3-8 \mathrm{keV}$ energy range, assuming the Band model as the best-fit spectrum. In this figure, the time origin was set $10 \mathrm{~s}$ before the nominal trigger time, to show the weak, untriggered precursor. This has no effect on the determination of the afterglow decay slope, due to the late beginning of the XRT observation.

column density $N_{\mathrm{H}}$ and for the photon index $\Gamma$ did not show significant variations between the first (WT data) and second part (PC data) of the observation. In fact, combining the data from the two segments together, we obtained an excellent $\chi^{2}$ value, indicating that the spectral properties of the afterglow did not change during the observation. Figure 4 shows the XRT spectrum (filled circles), together with the best-fit absorbed powerlaw model (only data simultaneous with the XMM-Newton observation were used for the plot; see Sect. 5).

To look for the presence of absorbing material in the proximity of the afterglow, we tried to estimate the Galactic hydrogen column density $N_{\mathrm{H}, \mathrm{MW}}$ towards the GRB direction. We found three different measurements: Dickey \& Lockman (1990) give $N_{\mathrm{H}, \mathrm{MW}}=4.6 \times 10^{20} \mathrm{~cm}^{-2}$; the Leiden/Argentine/Bonn Galactic H I Survey (Kalberla et al. 2005) provides a lower value, $N_{\mathrm{H}, \mathrm{MW}}=3.8 \times 10^{20} \mathrm{~cm}^{-2}$; last, the dust maps by (Schlegel et al. 1998) give $A_{\mathrm{V}}=0.12 \mathrm{mag}$, which corresponds to $N_{\mathrm{H}, \mathrm{MW}}=$ $2.2 \times 10^{20} \mathrm{~cm}^{-2}$ after assuming the prescription given by Predehl $\&$ Schmitt (1995). While the average of these three independent measurements is $(3.5 \pm 1.7) \times 10^{20} \mathrm{~cm}^{-2}$, we conservatively adopted the largest of the above values $\left(4.6 \times 10^{20} \mathrm{~cm}^{-2}\right)$. Stratta et al. (2004) estimated that the typical error affecting the maps by Dickey \& Lockman (1990) is 30\%, which is not far from the scatter among the three measurements. The best-fit hydrogen column density derived by the XRT afterglow spectrum is marginally unconsistent with the Galactic value. Fixing $N_{\mathrm{H}}=N_{\mathrm{H}, \mathrm{MW}}$ provided a poor fit $\left(\chi_{v}^{2}=1.20\right.$ for 39 d.o.f. $)$. The probability of such a decrease in the fit statistic is $<7.5 \%$, as estimated by an F-test. Therefore the XRT data marginally suggest the presence of additional absorbing material, likely located in the GRB rest frame. In the next section, we will present
Table 1. Best-fit spectral parameters for the two segments of the XRT observation (WT and PC data), fitted both separately and together. We report the results either leaving the hydrogen column density as a free parameter, or freezing it to the Galactic value.

\begin{tabular}{lccc}
\hline \hline & $N_{\mathrm{H}}\left(10^{21} \mathrm{~cm}^{-2}\right)$ & $\Gamma$ & $\chi_{v}^{2}\left(\chi^{2} /\right.$ d.o.f. $)$ \\
\hline XRT (WT) & $1.3_{-0.6}^{+0.7}$ & $1.92_{-0.24}^{+0.27}$ & $1.10(29.7 / 27)$ \\
XRT (PC) & $1.4_{-0.7}^{+0.8}$ & $2.00_{-0.31}^{+0.38}$ & $1.05(12.6 / 12)$ \\
XRT (WT+PC) & $1.4_{-0.5}^{+0.6}$ & $1.95_{-0.21}^{+0.21}$ & $1.10(42.0 / 38)$ \\
\hline XRT (WT) & 0.46 (frozen) & $1.58 \pm 0.12$ & $1.22(34.1 / 28)$ \\
XRT (PC) & 0.46 (frozen) & $1.58 \pm 0.14$ & $1.41(19.1 / 13)$ \\
XRT (WT+PC) & 0.46 (frozen) & $1.61 \pm 0.10$ & $1.20(47.0 / 39)$ \\
\hline
\end{tabular}

further evidence for the presence of excess absorption, based on $\mathrm{XMM}-$ Newton data with a better $\mathrm{S} / \mathrm{N}$ ratio.

\section{XMM-Newton data analysis and results}

The afterglow of GRB 050326 was also observed by XMM-Newton as a target of opportunity, starting on 2005 Mar. 26 at 18:25 UT (8.5 $\mathrm{h}$ after the burst). The observation lasted for $45.8 \mathrm{ks}$. Data were collected with the European Photon Imaging Camera (EPIC), which consists of the PN (Strüder et al. 2001) and of two MOS detectors (Turner et al. 2001). All the cameras were operated in full-frame mode with a thin and medium optical filter on PN and MOS, respectively. A preliminary analysis of these data was presented by De Luca et al. (2005a). 


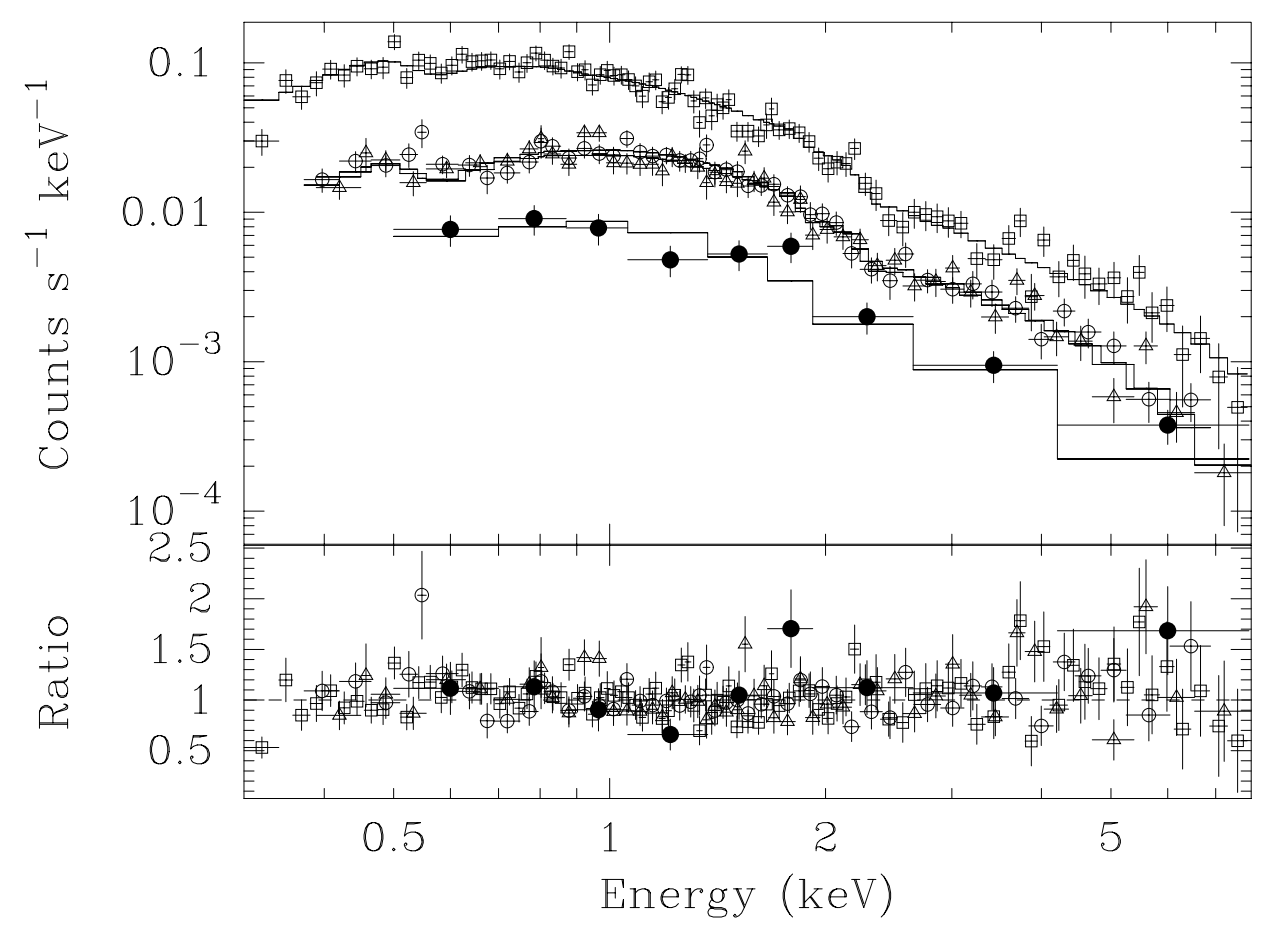

Fig. 4. The spectra of the GRB 050326 afterglow as observed by XMM-Newton (PN: squares; MOS1: open circles; MOS2: triangles) and by Swift-XRT (filled circles). The solid lines represent the best-fit absorbed power-law model convolved with the instrumental responses (see Table 2 for the best-fit parameters). XRT data were selected from the time interval covered by the XMM-Newton observation.

\subsection{Data and temporal analysis}

The appropriate observation data files were retrieved from the XMM Science Archive. The data reduction was performed using the most recent release of the XMM Science Analysis Software (SAS v6.1.0), with the standard pipeline tasks (epproc and emproc for PN and MOS, respectively). The observation was badly affected by high particle background (soft proton flares), with almost no nominal (quiescent) background time intervals. The back-illuminated PN CCD is particularly sensitive to this background; indeed, more than $25 \%$ of the PN observing time was lost due to the detector switching to its counting mode ${ }^{1}$. Nonethless the afterglow of GRB 050326 (source XMMU J002748.8-712217; Ehle \& Perez Martinez 2005) was clearly detected in all cameras. The astrometry of the EPIC images was improved by cross-correlating serendipitous X-ray sources in the field with objects in the USNO-B1 catalog. This yielded the following refined coordinates for the afterglow: $\alpha_{\mathrm{J} 2000}=00^{\mathrm{h}} 27^{\mathrm{m}} 49 !^{\prime} 1, \delta_{\mathrm{J} 2000}=-71^{\circ} 22^{\prime} 16^{\prime \prime} \cdot 3$, with a $1-\sigma$ uncertainty of 1 .' 5 . The EPIC and XRT positions differ by $11^{\prime \prime} 7$, and are therefore fully consistent within the uncertainties.

In order to retain a $\mathrm{S} / \mathrm{N}$ ratio large enough to perform the temporal and spectral analysis, a standard time-filtering approach to screen soft proton flares could not be applied (a high particle flux was present during the whole observation). Thus, source events were extracted with a particularly stringent spatial selection, considering only the innermost portion of the point spread function. We used a circle of $15^{\prime \prime}$ radius (containing $\approx 65 \%$ of the EEF). The PSF correction was applied to the flux and spectral measurements by computing the ad-hoc effective area using the SAS task arfgen. The error in this procedure is

\footnotetext{
1 The counting mode is activated when the count rate in a quadrant exceeds the telemetry limit ( $\sim 00$ count $\mathrm{s}^{-1}$ for the PN). In this mode, the information for individual events of that quadrant are not transmitted to ground.
}

estimated to be at most at the $5 \%$ level $^{2}$ and it was properly taken into account in the light curve error budget.

Background events were selected from source-free regions within the same CCD chip where the source was imaged. In particular, for the PN data we used 2 boxes of $45^{\prime \prime} \times 25^{\prime \prime}$ located at the same distance from the readout node as the target; for the MOS we used an annulus centered at the target position with inner and outer radii of $90^{\prime \prime}$ and 180", respectively. With such a choice, the background amounted to $\sim 13 \%$ and $\sim 9 \%$ of the counts in the source extraction region for PN and MOS data, respectively, in the $0.3-8 \mathrm{keV}$ range. The overall (backgroundsubtracted) number of source events was 3990, 1850 and 1760 in the PN, MOS1 and MOS2 detectors, respectively.

The background-subtracted count rate clearly showed a declining trend with time. We again fitted the light curve assuming a power law decay. The value of the decay slope $\alpha$ was evaluated independently using PN, MOS1 and MOS2 data, yielding fully consistent results. We therefore repeated the fit using the combined dataset, finding $\alpha=1.72 \pm 0.09$ in the $0.3-8 \mathrm{keV}$ energy range $\left(\chi_{v}^{2}=1.30\right.$ for 40 d.o.f.). The background-subtracted light curve is shown in Fig. 3, together with the XRT light curve, after converting the count rates to fluxes using the best-fit absorbed power-law models described in Sects. 3.3 and 4.2.

\subsection{Spectral analysis}

The source and background spectra were extracted from the same regions used for the temporal analysis, as described above. The source spectra were binned in order to have at least 30 counts per energy bin and to oversample the instrumental energy resolution by a factor 3 . Ad-hoc response matrices and effective area files were created with the SAS tasks rmfgen and

\footnotetext{
2 See page 9 of http://xmm.vilspa.esa.es/docs/documents/ CAL-TN-0018-2-4.pdf
} 
Table 2. The spectral parameters as measured separately by XMM-Newton and XRT, fitting the data both separately and together. For this fit, XRT data were selected from the same time interval covered by the XMM-Newton observation. Due to the limited statistics, $N_{\mathrm{H}}$ was frozen to the value derived by XMM-Newton when fitting XRT data. The errors are at $90 \%$ confidence level for a single parameter of interest.

\begin{tabular}{lccc}
\hline \hline Instrument & $N_{\mathrm{H}}\left(10^{21} \mathrm{~cm}^{-2}\right)$ & $\Gamma$ & $\chi_{v}^{2}\left(\chi^{2} /\right.$ d.o.f. $)$ \\
\hline EPIC & $1.3_{-0.1}^{+0.1}$ & $2.13_{-0.06}^{+0.06}$ & $1.17(222.5 / 190)$ \\
XRT & 1.3 (frozen) & $1.96_{-0.27}^{+2.29}$ & $1.43(11.4 / 8)$ \\
EPIC + XRT & $1.3_{-0.1}^{+0.1}$ & $2.09_{-0.08}^{+0.05}$ & $1.18(219.7 / 197)$ \\
\hline
\end{tabular}

arfgen, respectively. The spectral analysis was performed using XSPEC (v11.3). The spectra were fitted simultaneously in the $0.3-8 \mathrm{keV}$ band. Since the MOS observation started $\approx 1 \mathrm{~h}$ earlier than the PN, a PN/MOS normalization factor was introduced in the fit as a further free parameter. Due to the fading of the source, this also implies that the observed time-averaged flux is expected to be higher in the MOS than in the PN.

An absorbed power-law model reproduced the spectrum quite well $\left(\chi_{v}^{2}=1.17\right.$ for 190 d.o.f.). The best-fit parameter values are reported in Table 2 (first row); the MOS-PN normalization factor was $1.08 \pm 0.04$. Figure 4 shows the spectra collected by the EPIC cameras together with the best-fit model. Both the photon index and the hydrogen column density are in good agreement with those found by XRT, but are much better constrained. In particular, the value of $N_{\mathrm{H}}$ inferred from the fit was significantly larger than the Galactic one $N_{\mathrm{H}, \mathrm{MW}}$ (Sect. 3.3). Moreover fixing $N_{\mathrm{H}}=N_{\mathrm{H}, \mathrm{MW}}$ resulted in a much poorer fit $\left(\chi_{v}^{2}=1.85\right.$ for 191 d.o.f.), and even increasing $N_{\mathrm{H}, \mathrm{MW}}$ by $30 \%$ (see Sect. 3.3 and Stratta et al. 2004) the fit was still unacceptable $\left(\chi_{v}^{2}=1.65\right.$ for 191 d.o.f.). A significant improvement was achieved by fixing $N_{\mathrm{H}}=N_{\mathrm{H}, \mathrm{MW}}$ and adding to the spectral model an extra neutral absorber at redshift $z$ with column density $N_{\mathrm{H}, z}$. This yielded $\chi_{v}^{2}=1.03$ (189 d.o.f.); the chance probability of such improvement, as estimated by an F-test, was $<1 \times 10^{-6}$ with respect to the model containing only one absorbing component (with free $N_{\mathrm{H}}$ ). After adding the extra absorption component, the best-fit power law photon index was $\Gamma=2.03 \pm 0.05$, while the intrinsic gas column density and the redshift were $N_{\mathrm{H}, z} \sim 6 \times 10^{22} \mathrm{~cm}^{-2}$ and $z \sim 6$, respectively. However, the latter two values are not well constrained, owing to their strong correlation (Fig. 5). In any case, the spectral fit allowed us to constrain $N_{\mathrm{H}, z}>4 \times 10^{21} \mathrm{~cm}^{-2}$ and $z>1.5$ (at $90 \%$ confidence level for 2 parameters of interest; see inset in Fig. 5). We investigated the dependence of these confidence contours on the assumed value of the Galactic column density. By varying $N_{\mathrm{H}, \mathrm{MW}}$ by $50 \%$ (within the range discussed above), we found that the $90 \%$ confidence interval on $N_{\mathrm{H}, z}$ varies by $20 \%$, while that on $z$ varies by less than $10 \%$.

The observed (time-averaged) fluxes in the $0.2-10 \mathrm{keV}$ band were $\sim 5.1 \times 10^{-13}$ and $\sim 5.5 \times 10^{-13} \mathrm{erg} \mathrm{cm}^{-2} \mathrm{~s}^{-1}$ in the PN and MOS, respectively. The corresponding unabsorbed fluxes were $\sim 7.4 \times 10^{-13}$ and $\sim 7.9 \times 10^{-13} \mathrm{erg} \mathrm{cm}^{-2} \mathrm{~s}^{-1}$.

In order to search for possible line features in the spectrum (both in emission and in absorption) we divided the $0.4-5 \mathrm{keV}$ energy range into $0.2 \mathrm{keV}$ intervals. For the continuum, we assumed the best-fit absorbed power-law model (including also the rest-frame column density). For each of the intervals, we added a Gaussian line with fixed width (smaller than the instrumental resolution) and central energy free to vary within the selected interval; the normalization could be either positive or negative. We

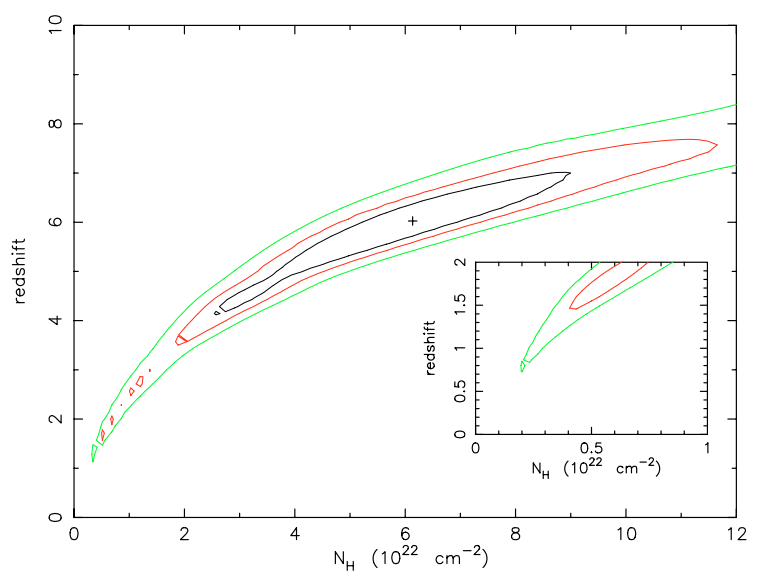

Fig. 5. Confidence contours $(68 \%, 90 \%$ and $99 \%$ levels for 2 parameters of interest) for the gas column density $N_{\mathrm{H}, z}$ and the redshift $z$ of the intrinsic absorber, as computed from the fit to the EPIC spectra. The Galactic column density was assumed to be $N_{\mathrm{H}, \mathrm{MW}}=4.6 \times 10^{20} \mathrm{~cm}^{-2}$ (Dickey \& Lockman 1990). The inset shows a zoom-in of the lowredshift region.

then repeated the exercise with different choices of the energy intervals. We found no significant lines in the $0.4-5 \mathrm{keV}$ range in the combined MOS/PN dataset. The upper limit (3- $\sigma$ ) on the equivalent width of any line is $\sim 50$ and $\sim 250 \mathrm{eV}$ in the $0.4-2$ and 2-5 keV energy ranges, respectively.

We also tried to fit the spectra with thermal models (e.g. Reeves et al. 2002; Lazzati 2003). A redshifted, optically thin plasma emission model (MEKAL in XSPEC) was used, with the redshift linked to that of the intrinsic absorber. The fit worsened $\left(\chi_{v}^{2} \approx 1.4\right)$ with respect to the simple power law model, either fixing the metal abundances to Solar values, or leaving them as free parameters.

Finally, we looked for possible spectral evolution with time, using the power law plus redshifted absorber model described above. For this study we divided the data into two subsets with exposure times of $\sim 15.8$ and $\sim 27.6 \mathrm{ks}$, each subset containing approximately half of the afterglow counts. We then extracted the corresponding spectra for the source and the background. No significant $(>3-\sigma)$ variations in the spectral parameters were found (except of course for the flux normalization).

\section{XMM-Newton/Swift-XRT comparison}

As noted above, the spectral parameters derived from using XRT and XMM-Newton data nicely agree within the errors. To better check the consistency between the afterglow temporal and spectral properties derived by the two satellites, we performed a more accurate operation. We selected XRT data from the MOS observing time interval (35-76 ks after the burst). During this period, the XMM-Newton observation was continuous, whereas Swift completed eight orbits, providing $13 \mathrm{ks}$ of effective exposure time for XRT. Therefore in order to compare the two datasets, we had to assume that both the spectrum and the light curve behaved in a regular fashion. For example, a flare during a Swift occultation would distort the results of the comparison. This assumption seems fully justified by the XMM-Newton data, which show a regular behaviour of the light curve without significant deviations from a power-law decay.

We fitted XRT data using the absorbed power law model. For the sake of comparison between the two instruments, we did not add any rest-frame absorption component. Due to the 
Table 3. Summary of optical and ultraviolet observations. All data but the point in the $R$ band were measured by Swift-UVOT. The $R$-band observations is by Tristram et al. (2005). All measurements were corrected for the Galactic extinction $\left(A_{\mathrm{V}}=0.123\right.$ mag; Schlegel et al. 1998), assuming the Milky Way extinction curve by Pei (1992). The optical-to-X-ray spectral index is $\beta_{\mathrm{OX}}=\log \left(F_{v}^{\mathrm{opt}} / F_{v}^{\mathrm{X}}\right) / \log \left(v_{\mathrm{X}} / v_{\mathrm{opt}}\right)$. The X-ray frequency was set to $v_{\mathrm{X}}=1.5 \mathrm{keV}$, the logarithmic mean of the XRT observing range. The X-ray flux was computed by interpolating the XRT light curve to the time of the optical limit. The last column reports the ratio of the extrapolated X-ray flux to the optical one (assuming no spectral breaks between the two bands; see Fig. 7).

\begin{tabular}{lllllllll}
\hline \hline $\begin{array}{l}\text { Time since burst } \\
(\mathrm{s})\end{array}$ & Filter & $\begin{array}{l}\text { Wavelength } \\
(\AA)\end{array}$ & $\begin{array}{l}\text { Exposure time } \\
(\mathrm{s})\end{array}$ & $\begin{array}{l}\text { Extinction } \\
(\mathrm{mag})\end{array}$ & Magnitude & $\begin{array}{l}\text { Flux density } \\
(\mu \mathrm{Jy})\end{array}$ & $\beta_{\text {Ox }}$ & X-ray/optical ratio \\
\hline 3300 & $U V W 2$ & 1930 & 10 & 0.29 & $>17.41$ & $<99.11$ & $<0.52$ & $>23_{-6.4}^{+8.9}$ \\
3306 & $V$ & 5460 & 100 & 0.12 & $>18.66$ & $<107.5$ & $<0.45$ & $>65_{-21}^{+31}$ \\
3350 & $U V M 2$ & 2220 & 100 & 0.36 & $>18.54$ & $<34.17$ & $<0.32$ & $>75_{-21}^{+30}$ \\
3470 & $U V W 1$ & 2600 & 100 & 0.27 & $>18.75$ & $<27.80$ & $<0.29$ & $>103_{-30}^{+43}$ \\
5219 & $U$ & 3450 & 750 & 0.20 & $>19.65$ & $<11.00$ & $<0.24$ & $>176_{-54}^{+77}$ \\
9390 & $B$ & 4350 & 714 & 0.16 & $>20.87$ & $<17.47$ & $<0.46$ & $>52_{-16}^{+24}$ \\
\hline 24840 & $R_{\text {MOA }}$ & 6399 & 600 & 0.10 & $>20.20$ & $<25.70$ & $<0.74$ & $>10_{-3.4}^{+5.0}$ \\
\hline
\end{tabular}

limited statistics we fixed the column density to the value found by XMM-Newton (which is fully consistent with that found by XRT for the full observation). The results of the fit are presented in Table 2. Within the errors, the photon index is consistent with that found by XMM-Newton. Lastly we fitted together the XRT dataset and the XMM-Newton data from the three EPIC instruments (leaving $N_{\mathrm{H}}$ as a free parameter), obtaining our final best fit (Table 2).

Our second step was to compare the flux normalization factors for the two instruments. To this extent, we selected the data only from exactly overlapping intervals $(8.5 \mathrm{ks}$ effective observation time). We froze $N_{\mathrm{H}}$ and $\Gamma$ to the values found previously (which rest on a better statistics), computing only the normalization factors (the ratio of the fluxes observed by Swift-XRT and the EPIC detectors). The fit provided $0.90 \pm 0.14$ for MOS1, $0.95 \pm 0.16$ for MOS2, and $0.89 \pm 0.15$ for PN. This result indicate that, within the errors, the XRT current absolute flux calibration is good, providing perhaps slightly underestimated values (at the $\sim 5 \%$ level).

As shown in the previous sections, the light curves from XRT and XMM-Newton were well described by power laws with slopes $1.64 \pm 0.07$ and $1.72 \pm 0.09$ respectively. These values are consistent within their errors. Moreover, selecting XRT data from the $8.5 \mathrm{ks}$ time interval with simultaneous XMM-Newton observations, we found a slope $\alpha=1.73 \pm 0.12$, in perfect agreement with the XMM-Newton result (and consistent with that measured by XRT for the whole observation).

In order to directly compare the fluxes measured from the two satellites, we converted the $0.3-8 \mathrm{keV}$ count rates to unabsorbed fluxes in the same band (Fig. 3). To compute the conversion factor for the XMM-Newton spectrum, we used the absorbed (one component) power law model with the parameters reported in Table 2 (first row). For XRT, we calculated a conversion factor for each of the two operational modes, using in both cases the absorbed power law best-fit model (leaving $N_{\mathrm{H}}$ as a free parameter), as reported in Table 1 (first and second rows). The combined fit of the joint XRT and XMM data provided $\alpha=1.70 \pm 0.05$ (Fig. 3). In the following, we will adopt this value as the best determination of the temporal decay slope.

\section{Optical and ultraviolet observations}

The UVOT instrument onboard Swift observed the field of GRB 050326 together with XRT, starting 54 min after the trigger. In the subsequent orbits, it collected a series of images in its 6 broad-band filters ( $V, B, U, \mathrm{UVW} 1, \mathrm{UVM} 2$, and UVW2; Table 3 ). The afterglow was not detected in any of the single or coadded exposures. Summing the images in each of the six filters, we estimated the 3- $\sigma$ upper limits using the UVOT dedicated software (task uvotsource). The counts were extracted from a $6^{\prime \prime}$ and $12^{\prime \prime}$ radius aperture for the optical and ultraviolet filters respectively, after subtracting the background. We then corrected the upper limits for Galactic absorption (Schlegel et al. 1998), assuming the extinction curve of Pei (1992). Our final limits are summarized in Table 3 . With respect to the original values reported by Holland et al. (2005), our measurements were obtained adopting the most recent in-flight calibration.

The only reported ground-based optical observation for this burst was an $R$-band upper limit provided by the $0.6 \mathrm{~m}$ telescope at the Mt. John Observatory (Tristram et al. 2005). This measurement is also listed in Table 3.

\section{Discussion}

\subsection{The X-ray light curve}

To date, Swift has observed X-ray emission from dozens of GRB afterglows. A systematic analysis of their light curves has revealed several common features (Nousek et al. 2005; Chincarini et al. 2005). During the first few hundred seconds, a steep decay is often observed ( $\alpha \approx 3-5$; Tagliaferri et al. 2005a), usually interpreted as the tail emission from the prompt GRB (e.g. Cusumano et al. 2006a). This phase is followed by a much flatter decline ( $\alpha \approx 0-0.7$; e.g. Campana et al. 2005; Nousek et al. 2005 ), lasting up to $10^{3}-10^{5} \mathrm{~s}$ (and in some cases even longer). Then the light curve steepens again, leading to $\alpha \approx 1-1.5$; this phase was the one seen by BeppoSAX, XMM-Newton and Chandra. At late times, a further steepening is sometimes observed (e.g. Vaughan et al. 2006), likely the signature of a jetted outflow (Rhoads 1999). In some cases bumps and flares appear superimposed to the power-law decay, for up to several tens of ks after the prompt GRB emission (e.g. Burrows et al. 2005b).

The light curve of GRB 050326 exhibited a different behaviour with respect to that outlined above. Its light curve showed a single, unbroken decay from $\approx 55 \mathrm{~min}$ to $\approx 4.2 \mathrm{~d}$. However our coverage began relatively late, so that we may have missed early deviations from the power law behaviour.

In order to investigate the early stages of the afterglow and to analyze the connection between the prompt and afterglow emission, we extrapolated the afterglow flux to the time of the prompt 
emission. We then compared the obtained value with that expected from the prompt emission in the XRT band $(0.3-8 \mathrm{keV})$, computed adopting the Band best-fit model. The result is shown in Fig. 3, where the light circles indicate the prompt emission fluxes. Since the GRB spectrum is known in good detail (particularly since no breaks are expected between the BAT and XRT ranges), the extrapolation process should be quite reliable. As can be seen, if no temporal breaks were present in the X-ray light curve, the afterglow flux in the X-ray range exceeded the prompt one by a factor of $\sim 100$ (with a small uncertainty, due the tiny error in the decay index). We cannot exclude that such emission was present (since we have no prompt observations in the X-ray band), but, if present, the present component would appear as a very bright, soft excess. Such feature would not be unprecedented (Vanderspek et al. 2004; Vetere et al. 2006), but in this case it would likely contaminate the low-frequency end of the BAT spectrum. Moreover the soft excesses always contained less energy that the GRB proper. Thus the most conservative hypothesis is to assume that a break was present in the early light curve, or that the afterglow onset was delayed. Indeed as mentioned above, most of Swift afterglows show a shallow decline phase during the first thousands seconds after the GRB. Independently of any extrapolation, we note that GRB 050326 was distinctly different from most bursts observed by BeppoSAX, for which the backward extrapolation of the latetime X-ray afterglow roughly matched the prompt emission level in the X-ray range, as measured by the Wide Field Cameras (Frontera et al. 2000).

We also performed a different operation. Using the best-fit X-ray spectrum, we extrapolated the XRT flux to the BAT energy range (20-150 keV), and reported it at the time of the burst using the afterglow decay law. Also in this case, the expected value exceeded the observed prompt emission, but by a smaller factor. This again suggests that a break in the light curve was present before the beginning of the XRT observation, but the evidence is less compelling. For example, we cannot even exclude that the afterglow spectrum had a break between the XRT and BAT ranges, so that the extrapolation actually overestimated its flux.

\subsection{Constraints on the afterglow parameters}

The properties of the explosion can be inferred in the context of the standard afterglow model (e.g. Mészáros \& Rees 1997; Sari et al. 1998). In this context, the observed emission is due to synchrotron radiation from a decelerating relativistic shock, which produces a decaying flux with a power-law spectrum. Depending on the model parameters, definite relations between the spectral and temporal indices $\alpha$ and $\beta$ are predicted. The combined XMM-Newton and Swift-XRT data provide $\alpha=1.70 \pm 0.05$ and $\beta=1.09 \pm 0.08$. Both values are not unusual among GRB afterglows at comparable epochs (e.g. De Pasquale et al. 2005; Chincarini et al. 2005; Nousek et al. 2005). These numbers are consistent with a spherical outflow expanding inside a homogeneous medium, if the XRT range was between the injection and cooling frequencies ( $v_{\mathrm{i}}$ and $v_{\mathrm{c}}$, respectively). In this case, the model prediction is $\alpha=3 \beta / 2=1.63 \pm 0.12$, in excellent agreement with the measured value $\alpha=1.70 \pm 0.05$. All other possibilities (a wind-stratified medium, or a different location of the break frequencies) are excluded at $>3.5-\sigma$ level. The power-law index of the electron energy distribution is $p=1+4 \alpha / 3=1+2 \beta$, so that $p=3.25 \pm 0.06$. Such value is rather high, but not unprecedented.

No break was observed in the X-ray light curve of GRB 050326 between $55 \mathrm{~min}$ and $\sim 4.2 \mathrm{~d}$ after the burst. The condition $v_{\mathrm{i}}<v<v_{\mathrm{c}}$ thus held during this time range. While $v_{\mathrm{i}}$ typically lies below $\sim 10^{15} \mathrm{~Hz}$ for $t>1 \mathrm{~h}$ (e.g. Sari et al. 1998; Panaitescu \& Kumar 2000), keeping $v<v_{\mathrm{c}}$ up to $t>4.2 \mathrm{~d}$ requires $n_{0} \varepsilon_{\mathrm{B},-2}^{3 / 2}<3 \times 10^{-5} E_{\text {iso,54 }}^{-1 / 2}$, where $n=n_{0} \times 1 \mathrm{~cm}^{-3}$ is the ambient particle density, $\varepsilon_{\mathrm{B}}=10^{-2} \varepsilon_{\mathrm{B},-2}$ is the magnetic field energy fraction, and $E_{\text {iso }}=E_{\text {iso, } 54} \times 10^{54}$ erg is the (isotropicequivalent) fireball energy. This condition is difficult to satisfy (e.g. Panaitescu \& Kumar 2001; Yost et al. 2003), so it may be regarded as a problem for the model. We note however that for this burst both $\alpha$ and $\beta$ could be measured with good accuracy, so the consistency between the predicted and observed value of the decay index is remarkable.

The absence of any break also poses some constraints on the geometry of the emission. GRB afterglow light curves often show a late-time steepening, commonly interpreted as the result of a jetted geometry. For GRB 050326, this break likely occurred after the end of the Swift observations. In fact, breaks earlier than $\sim 1 \mathrm{~h}$ are usually due to different reasons (such as the end of the refreshed shock episode; e.g. Zhang et al. 2005). If interpreted as a jet break, a very narrow jet would be implied. Moreover, the decay slope in the monitored time range is quite flat compared with that expected (and usually observed) after jet breaks (e.g. Israel et al. 1999; Harrison et al. 2001; Klose et al. 2004). The measured decay would imply a hard electron distribution $(p<2)$. Using the relations provided by Dai \& Cheng (2001), an unreasonably low $p=4 \alpha-6=0.8 \pm 0.2$ would result (with the X-ray band being above $v_{\mathrm{c}}$ ), which would give rise to a spectrum completely inconsistent with the observed one. Assuming $v_{\mathrm{c}}$ above the observed range would only worsen the situation.

Assuming that the jet break occurred at $t_{\mathrm{b}} \gtrsim 4 \mathrm{~d}$ allows us to put some constraints on the jet opening angle and on the beaming-corrected gamma-ray energy. To this extent, the main obstacle is the lack of a well constrained redshift. Our spectral analysis of the XMM-Newton data allowed us only to set a broad range of $1.5<z<8$ (90\% confidence level) on the redshift. Using the bolometric fluence $\mathcal{F}$ of the prompt emission, the lower limit on $z$ provides a constraint on the GRB radiated energy:

$E_{\gamma, \text { iso }}=4 \pi \frac{D_{\mathrm{L}}^{2}(z)}{1+z} \mathcal{F}>1.4 \times 10^{53} \mathrm{erg}$,

where $D_{\mathrm{L}}>3.40 \times 10^{28} \mathrm{~cm}$ is the luminosity distance. GRB 050326 was therefore quite likely bright in gamma rays compared to other GRBs detected by Swift (e.g. Chincarini et al. 2005).

Following Sari et al. (1999), the jet half-opening angle can be constrained as follows:

$\vartheta_{\mathrm{j}}>6.7^{\circ}\left(\frac{1+z}{2.5}\right)^{-3 / 8}\left(\frac{E_{\gamma, \text { iso }}}{10^{53} \mathrm{erg}}\right)^{-1 / 8}\left(\frac{\eta}{0.2} \frac{n}{1 \mathrm{~cm}^{-3}}\right)^{1 / 8}$,

where $\eta$ is the prompt radiative efficiency and we have assumed $t_{\mathrm{b}}>4 \mathrm{~d}$. Note that the dependance from the gamma-ray energy and on the other parameters is rather mild. The corresponding beaming-corrected gamma-ray energy is then

$E_{\gamma, \mathrm{j}}=\frac{\pi}{2} \vartheta_{\mathrm{j}}^{2} E_{\gamma, \text { iso }} \propto\left(\frac{E_{\gamma, \text { iso }}}{1+z}\right)^{3 / 4} \propto \mathcal{F}^{3 / 4}\left(\frac{D_{\mathrm{L}}}{1+z}\right)^{3 / 2}$.

The quantity $D_{\mathrm{L}} /(1+z)$ has only a mild dependence upon $z$. For the redshift range allowed by our X-ray measurements $(1.5<$ $z<8)$, we have $1.4 \times 10^{28} \mathrm{~cm}<D_{\mathrm{L}} /(1+z)<2.8 \times 10^{28} \mathrm{~cm}$, so that $E_{\gamma, \mathrm{j}}>(3-8) \times 10^{51} \mathrm{erg}$. This is at the high end of the 


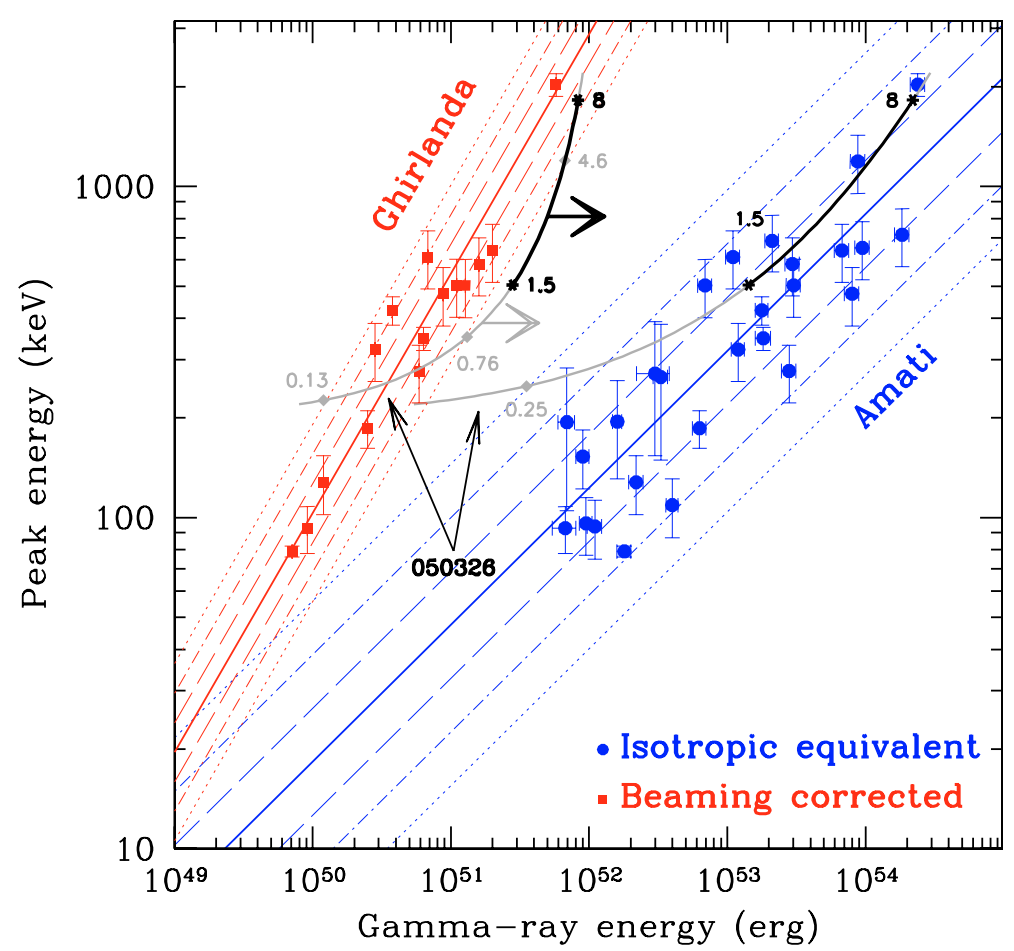

Fig. 6. Comparison of GRB 050326 with the Amati (right) and Ghirlanda (left) relations (Amati et al. 2002; Ghirlanda et al. 2004). The thick solid curves (black and grey) show the position of GRB 050326 as its redshift varies in the interval $0.1<z<10$. The Ghirlanda track is actually a boundary (as the horizontal arrows indicate), since we can infer only a lower limit to the beaming-corrected energy at each redshift. Filled circles and squares indicate the GRBs which define the above two relations, plotted as straight solid lines (together with their 1-, 2- and 3- $\sigma$ contours: long-dashed, dot-dashed and dotted lines, respectively). Data were taken from Ghirlanda et al. (2004, 2005). Grey diamonds indicate the intersection of the GRB 050326 tracks with the 3- $\sigma$ contours of the Amati and Ghirlanda relations. These points thus define the 3- $\sigma$ redshift ranges for which GRB 050326 was consistent with the two relations. In the two GRB 050326 tracks, the region $1.5<z<8$ (indicated by the X-ray data) is shown in black, bound by asterisks.

beaming-corrected gamma-ray energy distribution (Ghirlanda et al. 2004).

Figure 6 shows the position of GRB 050326 in the plane $E_{\gamma}$ vs. $E_{\mathrm{p}}$, to check how it compares with the Amati and Ghirlanda relations (Amati et al. 2002; Ghirlanda et al. 2004). As the redshift varies, the burst position follows the tracks indicated by the thick solid curves (see the figure caption for the details). The Ghirlanda track is actually a boundary, since the lower limit on $t_{\mathrm{b}}$ translates into a lower limit for $E_{\gamma, \mathrm{j}}$ for any given redshift. We can ask for which redshifts GRB 050326 was consistent with the two above relations. Only loose limits are provided by the Amati relation (which has a large dispersion): at the 3- $\sigma$ level, $z>0.25$ is implied. The comparison with the Ghirlanda relation is less solid, since further assumptions are needed (such as the ambient particle density and the break time). However, for our fiducial values, we have two allowed ranges: a low-redshift region $(0.1 \lesssim z \lesssim 0.8)$, plus a high-redshift solution $(z \gtrsim 4.5)$. A lower particle density would move the track towards the left, while a larger jet break time would shift it rightwards.

We note that the only way to make GRB 050326 in agreement with the Ghirlanda relation and simultaneously satisfy our constraints on the X-ray absorption $(z \gtrsim 1.5)$ is to require a high redshift for this event, since the low-redshift region is excluded by the fit to the X-ray column density at $>99 \%$ confidence level. Therefore although our arguments are rather speculative, and would surely need more conclusive data, we regard GRB 050326 as a moderate/high redshift candidate.

Recently, Liang \& Zhang (2005) have presented a modelindependent multidimensional correlation between the observed isotropic energy $E_{\gamma, \text { iso }}$, the rest-frame spectral peak energy $E_{\mathrm{p}}$, and the comoving break time $t_{\mathrm{b}} /(1+z)$. This relation, in principle, allows to compute $E_{\gamma \text {,iso }}$ (and $z$ ) for a GRB with known $E_{\mathrm{p}}$ and $t_{\mathrm{b}}$. However, no significant constraints could be inferred in the case of GRB 050326. Moreover, since this relation was derived using the break time as measured in the optical band, its application to X-ray data may not be valid (Liang \& Zhang 2005).

\subsection{Evidence for intrinsic absorption}

The presence of intrinsic absorption, besides allowing us to constrain the GRB redshift, has other important consequences. The rest-frame absorbing system has a hydrogen column density larger than $\sim 4 \times 10^{21} \mathrm{~cm}^{-2}$. For moderate redshifts, $N_{\mathrm{H}, z}$ would be much larger. Several afterglow observations, both from Swift and previous missions, showed evidence for excess Xray absorption in addition to the Galactic value (e.g. Galama \& Wijers 2001; Stratta et al. 2004; De Luca et al. 2005b). Recently, Campana et al. (2006) showed that about half of Swift afterglows have a large rest-frame column density, typical of giant molecular clouds (Reichart \& Price 2002). Given the connection between GRB explosion and supernovae (Galama et al. 1998; Stanek et al. 2003; Hjorth et al. 2003), this fact may constitute a powerful way to study the regions where massive star formation takes place in the high-redshift Universe (Ramirez-Ruiz et al. 2001). Lazzati \& Perna (2002) showed that the prompt GRB flux is able to ionize the surrounding medium up to radii as large as $\sim 5 \mathrm{pc}$, therefore leaving no absorbing material. Such process may have been observed in act for GRB 000528 (Frontera et al. 2004). The fact that a large column density was measured in GRB 050326 may imply that the absorbing material was 


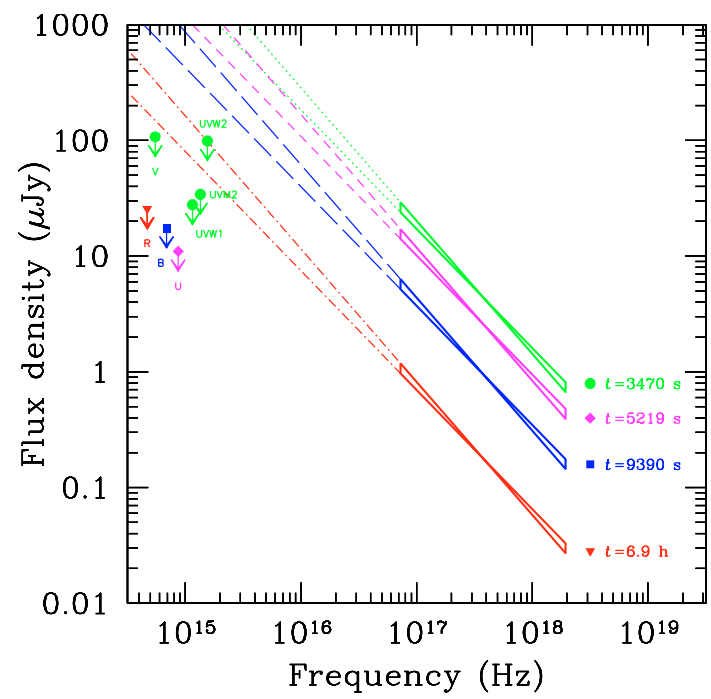

Fig. 7. Broad-band spectral energy distribution of the afterglow of GRB 050326, computed at different times (which are identified by different symbols). The shape of the X-ray spectrum was assumed to be constant throughout the observation, and the decay law was adopted to report the X-ray flux at the time of the optical measurements.

distributed over a wide region $(R \gtrsim 5 \mathrm{pc})$, or that the ionizing flux was not large.

For GRB afterglows, comparable absorption in the X-ray range is usually not accompanied by large extinction in the optical band (Galama \& Wijers 2001). Only small amounts of dust are usually inferred from the analysis of optical spectra, even when heavy extinction is observed in the X-ray afterglow. Several explanations were invoked to explain this discrepance, among which the destruction of dust from the burst and/or afterglow photons (Waxman \& Draine 2000), a large gas-to-dust ratio in the intervening material (Stratta et al. 2004), or an overabundance of $\alpha$ elements (Watson et al. 2005a). Unluckily, several factors hamper the study of this problem, such as the uncertaintes in the shape and normalization of the extinction curve, the possibility that GRBs occur in special, low-metallicity environments (Fynbo et al. 2003; McFadyen \& Woosley 1999), and in several cases the lack of the redshift determination.

For GRB 050326, no detection in the optical/ultraviolet band could be obtained. Table 3 reports the available upper limits to the afterglow flux, from both Swift-UVOT and ground-based observations. In Fig. 7 we show the optical-to-X-ray spectral energy distribution at different epochs. We computed the X-ray flux at the time of each available limit, adopting the decay law measured by Swift-XRT and XMM-Newton. Furthermore, no spectral evolution was assumed.

As it can be seen, the UVOT limits provide strong constraints, even if they are not particularly deep. In fact, GRB 050326 was bright in X-rays (with a flux of

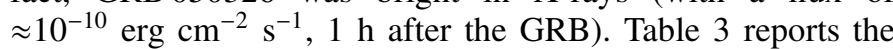
optical-to-X-ray spectral indices $\beta_{\text {OX }}$. For all our measurements, $\beta_{\mathrm{OX}}<\beta_{\mathrm{X}}$. Moreover, most of them violate the synchrotron limit $\beta_{\mathrm{OX}}>0.5$, which holds for a non-obscured synchrotron spectrum. GRB 050326 can therefore be classified as truly dark, according to the definition proposed by Jakobsson et al. (2004). This limit is quite robust, since no assumptions are made about the position of the synchrotron break frequencies. For this GRB we can go further in this line of reasoning. Our analysis of the temporal and spectral properties of the afterglow has indicated that the XRT range was below the cooling frequency $v_{\mathrm{c}}$. Therefore, the extrapolation of the XRT spectrum to the optical domain seems in this case reliable, since no spectral breaks are expected to lie between these two bands. This allows us to estimate the suppression factor suffered by the optical flux, and is reported in the last column of Table 3. Again large lower limits were found, implying conspicuous rest-frame extinction (up to a factor $\sim 100$ and more, corresponding to $>5 \mathrm{mag}$ ). The presence of the injection frequency $v_{\mathrm{i}}$ close to or blueward of the optical band may partly explain the flux dearth. However following the formulation of Panaitescu \& Kumar (2000), even choosing rather extreme parameters $\left(E_{\text {iso }}=10^{54} \mathrm{erg}, z=5\right.$, $\left.\varepsilon_{\mathrm{e}}=\varepsilon_{\mathrm{B}}=0.1\right), v_{\mathrm{i}}$ can at most be comparable to the ultraviolet observed frequencies. In particular at the time of the $U$-filter measurement, which provides the strongest constraint, $v_{\mathrm{i}}$ cannot be blueward of this band. So even if $v_{\mathrm{i}}$ has some role in this game, it cannot be responsible for the whole suppression of the optical flux. Moreover, low values of $\varepsilon_{\mathrm{B}}$ were required to keep the cooling frequency outside the XRT range (see Sect. 7.2), so that $v_{\mathrm{i}}$ was likely at much lower energies than the optical band.

The truly dark nature of this burst allows one of the following two possibilities. The burst may have suffered dust extinction in its host galaxy. The amount of dust is not straightforward to evaluate. The main obstacle is again the lack of the redshift, together with the unknown shape of the extinction curve. However our limit that more than 5 mag were missing in the observed $U$ band may roughly correspond to $A_{\mathrm{V}} \gtrsim 2$ mag for $z \sim 1.5$, even if many other solutions are acceptable. The second possibility is that GRB 050326 was at high redshift, as suggested by our analysis of the X-ray spectrum combined with the limits provided by the Ghirlanda relation. In this case virtually no flux is left blueward of the redshifted Lyman dropout. To suppress the flux in the $V$ band, $z \gtrsim 5$ would be required. However, the combination of a moderate redshift and mild absorption may relax this condition.

\section{Conclusions}

We have presented a detailed analysis of the GRB 050326 prompt and afterglow emission. The combined capabilities of Swift (which sampled the light curve for a relatively long time span) and XMM-Newton (which ensured very good statistics), allowed us to obtain a thorough characterization of the afterglow properties.

The prompt emission was relatively bright (with a $20-150 \mathrm{keV}$ fluence of $\sim 8 \times 10^{-6} \mathrm{erg} \mathrm{cm}^{-2}$ ). The spectrum was hard (photon index $\Gamma=1.25 \pm 0.03$ ), suggesting a peak energy at the high end of the BAT energy range or beyond. Indeed, thanks to the simultaneous detection of this burst by the Wind-Konus experiment (Golenteskii et al. 2005), the prompt spectrum could be fully characterized. The prompt bolometric fluence was $\mathcal{F} \sim 2.4 \times 10^{-5} \mathrm{erg} \mathrm{cm}^{-2}(1-10000 \mathrm{keV})$, and the observed peak energy was $E_{\mathrm{p}, \mathrm{obs}}=200 \pm 30 \mathrm{keV}$.

Due to pointing constraints, XRT and UVOT observations could start only $54 \mathrm{~min}$ after the GRB. The X-ray afteglow was quite bright, with a flux of $7 \times 10^{-11} \mathrm{erg} \mathrm{cm}^{-2} \mathrm{~s}^{-1}(0.3-8 \mathrm{keV})$ $1 \mathrm{~h}$ after the GRB. However no optical counterpart could be detected. The X-ray light curve showed a steady decline, with no breaks or flares. The best-fit power-law decay index was $\alpha=1.70 \pm 0.05$. Such regular behaviour is different from that usually observed by Swift, but this may be the result of the limited time coverage (observations could be carried out only between $54 \mathrm{~min}$ and $4.2 \mathrm{~d}$ after the burst). Indeed extrapolation of the afterglow light curve to the time of the prompt emission overpredicts the burst flux, and may suggest a slower decay before the beginning of the XRT observation. 
The analysis of the combined XRT and XMM-Newton data allowed to characterize in detail the afterglow spectrum. A fit with an absorbed power-law model provided a good description of the data, yielding a photon index $\Gamma=2.09 \pm 0.08$ and a column density significantly in excess of the Galactic value. The best-fit model was thus computed adding an extra absorption component, leaving its redshift $z$ free to vary. Although both $N_{\mathrm{H}, z}$ and $z$ could not be effectively constrained, a firm lower limit of $N_{\mathrm{H}, z}>4 \times 10^{21} \mathrm{~cm}^{-2}$ could be set. Therefore GRB 050326 adds to the growing set of afterglows with large rest-frame column density (Galama \& Wijers 2001; Stratta et al. 2004; Campana et al. 2006). The limits measured in the optical and ultraviolet region by UVOT lie well below the extrapolation of the X-ray spectrum. In particular they violate the synchrotron limit that the optical-to-X-ray spectral index should be larger than 0.5 . This implies a large extinction and/or a high redshift.

The X-ray spectral analysis also allowed us to set the lower limit $z>1.5$ to the redshift of the absorbing component (and therefore of the GRB). The isotropic-equivalent gamma-ray energy was then $E_{\gamma \text {,iso }}>1.4 \times 10^{53} \mathrm{erg}$. The temporal and spectral properties of the afterglow were nicely consistent with a spherical fireball expanding in a uniform medium, with the cooling frequency above the $\mathrm{X}$-ray range. We could therefore set a lower limit to the jet break time $t_{\mathrm{b}} \gtrsim 4 \mathrm{~d}$. The jet opening angle could be constrained to be $\vartheta_{\mathrm{j}} \gtrsim 7^{\circ}$, with only a weak dependence on the (unknown) fireball energy. The beaming-corrected gammaray energy was $E_{\gamma, \mathrm{j}}=(3-8) \times 10^{51}\left(t_{\mathrm{b}} / 4 \mathrm{~d}\right)^{3 / 4} \mathrm{erg}$, independently from the redshift. Thus GRB 050326 released a large amount of energy in the form of gamma rays (only GRB 990123 had a larger energy in the sample of Ghirlanda et al. 2004).

To be consistent with the Ghirlanda relation (Ghirlanda et al. 2004), two redshift ranges are allowed, either at low $(z \lesssim 0.8)$ or high $(z \gtrsim 4.5)$ redshift. However to simultaneously satisfy the limits derived from the X-ray spectral analysis, only the highredshift solution appears possible. Nonetheless we note that the Ghirlanda relation is based upon a small sample, so any inference about the redshift cannot yet be regarded as conclusive. However the results from the X-ray spectra, the consistency of the GRB 050326 properties with the Ghirlanda relation and the strong dearth of optical/ultraviolet afterglow flux, are consistent overall with a moderate/high redshift $(z \gtrsim 4)$ for this GRB. A search for the host galaxy through deep infrared and optical imaging may conclusively settle this issue.

Acknowledgements. This work is supported at OAB by the ASI grant I/R/039/04, at Penn State by the NASA contract NAS5-00136, and at the University of Leicester by the PPARC grant PPA/Z/S/2003/00507. We gratefully acknowledge the contributions of dozens of members of the XRT and UVOT teams at OAB, PSU, UL, GSFC, ASDC, and MSSL, and our subcontractors, who helped make this instrument possible. D.M. thanks INAF for financial support.

\section{References}

Amati, L., Frontera, F., Tavani, M., et al. 2002, A\&A, 390, 81 Band, D., Matteson, J., Ford, L., et al. 1993, ApJ, 413, 281

Barthelmy, S. D., Barbier, L. M., Cummings, J. R., et al. 2005, Space Sci. Rev., 120, 143

Berger, E., Kulkarni, S. R., Fox, D. B., et al. 2005, ApJ, 634, 501

Blustin, A. J., Band, D., Barthelmy, S., et al. 2006, ApJ, 637, 901

Burrows, D. N., Hill, J. E., Nousek, J. A., et al. 2005a, Space Sci. Rev., 120, 165

Burrows, D. N., Romano, P., Falcone, A., et al. 2005b, Science, 309, 1833

Campana, S., Antonelli, L. A., Chincarini, G., et al. 2005, ApJ, 625, L23
Campana, S., Romano, P., Covino, S., et al. 2006, A\&A, 449, 61

Cummings, J., Barthelmy, S., Barbier, L., et al. 2005, GCN Circ., 3145 Cusumano, G., Mangano, V., Angelini, L., et al. 2006a, ApJ, 639, 316 Cusumano, G., Mangano, V., Chincarini, G., et al. 2006b, Nature, 440, 164 Chincarini, G., Moretti, A., Romano, P., et al. 2005 [arXiv : astro-ph/0506453] Dai, Z. G., \& Chen, K. S. 2001, ApJ, 558, L109

De Luca, A., Götz, D., \& Campana, S. 2005a, GCN Circ., 3293 De Luca, A., Melandri, A., Caraveo, P., et al. 2005b, A\&A, 440, 85 De Pasquale, M., Piro, L., Gendre, B., et al. 2005, A\&A, submitted [arXiv: astro-ph/0507708]

Dickey, J. M., \& Lockman, F. J. 1990, ARA\&A, 28, 215

Ehle, M., \& Perez Martinez, R. 2005, GCN Circ., 3149

Frontera, F., Amati, L., Costa, E., et al. 2000, ApJ, 127, 59

Frontera, F., Amati, L., Lazzati, D., et al. 2004, ApJ, 614, 301

Fynbo, J. P. U., Jakobsson, P., Møller, P., et al. 2003, A\&A, 406, L63

Galama, T. J., Vreeswijk, P. M., van Paradijs, J., et al. 1998, Nature, 395, 670

Galama, T. J., \& Wijers, R. A. M. J. 2001, ApJ, 549, L209

Gehrels, N., Chincarini, G., Giommi, P., et al. 2004, ApJ, 611, 1005

Ghirlanda, G., Ghisellini, G., \& Lazzati, D. 2004, ApJ, 616, 331

Ghirlanda, G., Ghisellini, G., Lazzati, D., \& Firmani, C. 2005, Il Nuovo Cimento, in press [arXiv: astro-ph/0504184]

Golenteskii, S., Aptekar, R., Mazets, E., et al. 2005, GCN Circ., 3152

Haislip, J., Nysewander, M., Reichart, D., et al. 2006, Nature, 440, 181

Harrison, F., Yost, S. A., Sari, R., et al. 2001, ApJ, 559, 123

Hill, J. E., Burrows, D. N., Nousek, J. A., et al. 2004, Proc. SPIE, 5165, 175

Hjorth, J., Sollerman, J., Møller, P., et al. 2003, Nature, 423, 847

Holland, S. T., Still, M., Landsman, W., et al. 2005, GCN Circ., 3150

Israel, G. L., Marconi, G., Covino, S., et al. 1999, A\&A, 348, L5

Jakobsson, P., Hjorth, J., Fynbo, J. P. U., et al. 2004, ApJ, 617, L21

Jakobsson, P., Levan, A., Fynbo, J. P. U., et al. 2006, A\&A, 447, 897

Kalberla, P. M. W., Burton, W. B., Hartmann, D., et al. 2005, ApJ, 440, 775

Kawai, N., Kosugi, G., Aoki, K., et al. 2006, Nature, 440, 184

Klose, S., Greiner, J., Rau, A., et al. 2004, AJ, 128, 1942

Lazzati, D. 2003, A\&A, 399, 913

Lazzati, D., \& Perna, R. 2002, MNRAS, 330, 383

Liang, E., \& Zhang, B. 2005, ApJ, 633, 611

Markwardt, C., Barthelmy, S., Barbier, L., et al. 2005, GCN Circ., 3143

McFadyen, A. I., \& Woosley, S. E. 1999, ApJ, 524, 262

Mészáros. P., \& Rees, M. J. 1997, ApJ, 476, 232

Moretti, A., Campana, S., Romano, P., et al. 2005a, GCN Circ., 3147

Moretti, A., Campana, S., Mineo, T., et al. 2005b, Proc. SPIE, 5898, 360

Moretti, A., Perri, M., Capalbi, M., et al. 2006, A\&A, 448, L9

Nousek, J., Kouveliotou, C., Grupe, D., et al. 2005, ApJ, submitted [arXiv: astro-ph/0508332]

Panaitescu, A., \& Kumar, P. 2000, ApJ, 543, 66

Panaitescu, A., \& Kumar, P. 2001, ApJ, 560, L49

Pei, Y. C. 1992, ApJ, 395, 130

Predehl, P., \& Schmitt, J. H. M. M. 1995, A\&A, 293, 889

Price, P. A., Cowie, L. L., Minezaki, T., et al. 2005, ApJ, submitted [arXiv: astro-ph/0509697]

Ramirez-Ruiz, E., Trentham, N., \& Blain, A. W. 2001, MNRAS, 329, 465

Rhoads, J. E. 1999, ApJ, 525, 737

Reeves, J. N., Watson, D., Osborne, J. P., et al. 2002, Nature, 416, 512

Reichart, D. E., \& Price, P. A. 2002, ApJ, 565, 174

Roming, P. W. A., Kennedy, T. E., Mason, K. O., et al. 2005a, Space Sci. Rev., 120, 95 Roming, P. W. A., Schady, P., Fox, D. B., et al. 2005b, [arXiv: astro-ph/0509273]

Sari, R., Piran, T., \& Narayan, R. 1998, ApJ, 497, L17

Sari, R., Piran, T., \& Halpern, J. P. 1999, ApJ, 519, L17

Schlegel, D. J., Finkbeiner, D. P., \& Davis, M. 1998, ApJ, 500, 525

Spergel, D. N., Verde, L., Peiris, H. V., et al. 2003, ApJS, 148, 175

Stanek, K. Z., Matheson, T., Garnavich, P. M., et al. 2003, ApJ, 591, L17

Stratta, G., Fiore, F., Antonelli, L. A., Piro, L. \& De Pasquale, M. 2004, ApJ, 608, 846

Strüder, L., Briel, U., Dennerl, K., et al. 2001, A\&A, 365, L18

Tagliaferri, G., Goad, M., Chincarini, G., et al. 2005a, Nature, 436, 985

Tagliaferri, G., Antonelli, L. A., Chincarini, G., et al. 2005b, A\&A, 443, L

Tristram, P., Jelínek, M., de Ugarte-Postigo, A., et al. 2005, GCN Circ., 3151

Turner, M. J. L., Abbey, A., Arnaud, M., et al. 2005, A\&A, 365, L27

Vanderspek, R., Sakamoto, T., Barraud, C., et al. 2004, ApJ, 617, 1251

Vaughan, S., Goad, M. R., Beardmore, A. P., et al. 2006, ApJ, 638, 920

Vetere, L., Massaro, E., Costa, E., Soffitta, P., \& Ventura, G. 2006, A\&A, 447, 499

Watson, D., Fynbo, J. P. U., Ledoux, C., et al. 2005a, ApJ, submitted [arXiv: astro-ph/0510368]

Watson, D., Reeves, J. N., Hjorth, J., et al. 2005b, ApJ, in press [arXiv: astro-ph/0509640]

Waxman, E., \& Draine, B. T. 2000, ApJ, 537, 796

Yost, S. A., Harrison, F. A., Sari, R. \& Frail, D. A. 2003, ApJ, 597, 459

Zhang, B., Fan, Y. Z., Dyks, J., et al. 2005, ApJ, submitted [arXiv: astro-ph/0508321] 